\title{
Dichromatic spectral measurement circuit in vanilla CMOS
}

\author{
D. B. Fasnacht, T. Delbruck \\ Inst. of Neuroinformatics, UNI-ETH Zurich, Switzerland
}

\begin{abstract}
The circuit described in this paper uses a "vertacolor" stacked two-diode structure to measure relative long and short wavelength spectral content. The p-type source-drain to nwell forms the top diode and the nwell-psubstrate diode forms the bottom diode. The circuit output is a digital PWM signal whose frequency encodes absolute intensity and whose duty cycle encodes the relative photodiode current. This signal is formed by a self-timed circuit that alternately discharges the top and bottom photodiodes. This circuit was fabricated in a standard 3M $2 \mathrm{P} 0.5$ $\mu \mathrm{m}$ CMOS process. Monochromatic stimulation shows that the duty cycle varies between $50 \%$ and $7 \%$ as the photon wavelength is varied between $400 \mathrm{~nm}$ to $750 \mathrm{~nm}$. The output frequency is 150 $\mathrm{Hz}$ at incident irradiance of $1.7 \mathrm{~W} / \mathrm{m}^{2}$. Chip-to-chip variation of PWM duty cycle and frequency is about $1 \%$ measured over 5 chips. Power consumption is $20 \mu \mathrm{W}$. A modified version of this circuit could form the basis for simple color vision sensors built in widely-available vanilla CMOS.
\end{abstract}

\section{INTRODUCTION}

In common color imaging sensors, a patterned RGB color filter layer is placed above the silicon. Progress in developing novel color vision processing technology is greatly hampered by the cost and lack of availability of color filter technology. These standard color filters can only be applied at the wafer level [1], restricting development to niche processes not widely available on multi-project providers. Using the wellknown spectral separation capabilities [2] of stacked silicon photodiodes for color photography or video imaging is difficult and requires special CMOS processes [3] (somehow refer to foveon here). The possibility of using this capability given in standard CMOS processes for vision (rather than imaging) has not been widely explored.

In this work we are motivated by the observation that most animals are dichromats rather than trichromats and there is a high incidence (5-8\%) in humans of red-green color blindness [4]. Thus a basic separation into long vs. short wavelength must be sufficient for many vision tasks.

A dichromatic separation capability is available in every CMOS process because the stacked photodiodes that are formed by active inside well have different spectral sensitivities (Fig. 1). Here we call this a "vertacolor" photodiode structure. The average depth a photon penetrates the silicon before it is absorbed depends on its wavelength. The longer the wavelength, the deeper the photons penetrate the silicon. Thus the two types of photodiodes have a different spectral response (Fig. 2). The shallow diode is more sensitive to short wavelength light and the buried diode is more sensitive to longer wavelengths. The ratio of the two different photocurrents is a strong function of wavelength and can provide information about the color.

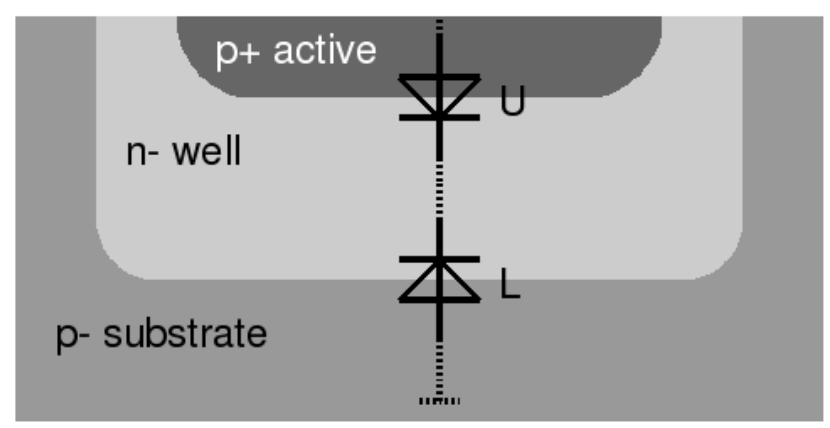

Fig. 1. Vertical cut through the silicon showing the stacked photodiodes (the vertacolor structure)

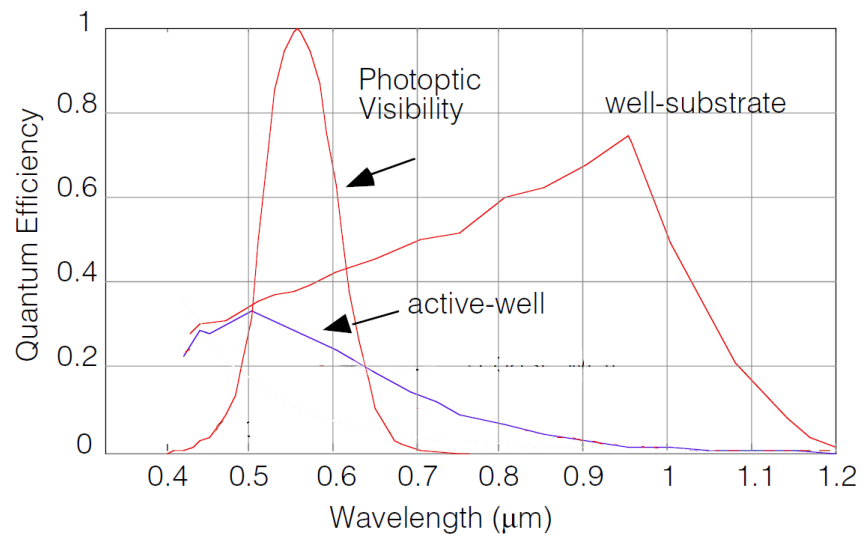

Fig. 2. Quantum efficiency (QE) for active and well photodiodes depends on photon wavelength (Adapted from a similar but not identical process. [5])

These stacked photodiodes form an upper pn junction between active and well and a lower pn junction between well and substrate. Because both diodes share the n-type region (well) and one node is "hardwired" to ground (substrate), it is hard to separate the two currents. The current of the upper diode is available at the active node. The only other available current is the current at the well node, which is the sum of both photocurrents.

If both currents were allowed flow continuously, the photocurrent of the lower diode would have to be "calculated" by somehow subtracting the upper current from the summed current. Because such a calculation is very mismatch-prone, we use a different approach to compute the ratio of the photocurrents.

To measure the ratio of photocurrents, we reverse bias both photodiodes and then let the photocurrent discharge the upper 


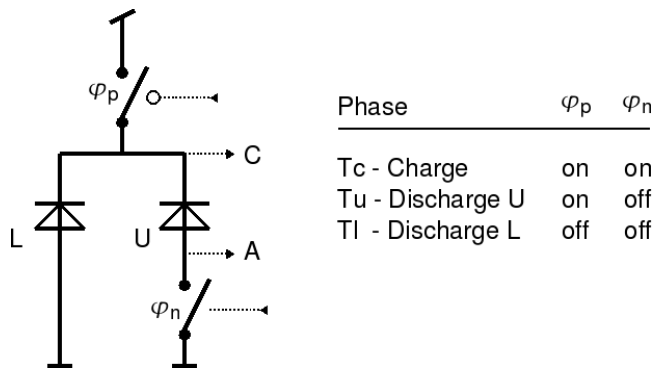

Fig. 3. Vertacolor structure and switch states

and then the lower diode. We output a signal that is low during the discharge of the upper diode and high during the discharge of the lower diode. The duty cycle of this signal contains the information we are looking for, the long versus short wavelength content of the incident light.

To generate this signal we use a self-timed control circuit.

\section{Circuit Details}

To be able to charge and let discharge the vertacolor structure, we need switches $\varphi_{n}$ and $\varphi_{p}$ to conditionally connect the diodes to the power rails. Fig. 3 shows these switches and defines the symbols $\mathrm{U}$ and $\mathrm{L}$ for the upper and lower diodes and $\mathrm{A}$ and $\mathrm{C}$ for the active and common nodes.

The three states the circuit takes are:

1) $T_{c}$ : when a reverse voltage is applied to charge both diode capacitances

2) $T_{u}$ : when the upper diode is discharged by photocurrent

3) $T_{l}$ : when the lower diode is discharged by photocurrent

These three states are repeated by the self-timed control circuit. Fig. 3 also shows how the switches are set in each state.

The charging duration $T_{c}$ is negligibly brief compared to $T_{u}$ and $T_{l}$. Thus the period $T=T_{u}+T_{l}$.

The PWM output signal is the signal controlling $\varphi_{p}$. It is low during $T_{u}$ (and also $T_{c}$ ), and high during $T_{l}$. The frequency $f=1 / T$ is proportional to the absolute light intensity.

The ratio of short to long wavelength photocurrents is $R=$ $T_{l} / T_{u}$ but the duty cycle $D=T_{l} / T=R /(R+1)$ contains the same information. The advantage of $D$ is that it can easily be computed in analog by an RC filter, unlike $R$.

\section{A. What happens during a cycle}

Fig. 4 shows the status of the switches and the change of the voltages $A$ and $C$ for each of the consecutive states. These are also shown in the scope traces in Fig. 7.

1) State $T_{c}$ : When $T_{c}$ is entered both diodes have previously been discharged. Now both switches are shorted. $A$ is thus grounded and $C$ is connected to $V_{D D}=5 \mathrm{~V}$. The current that now flows through $\varphi_{p}$ charges the diode capacitances, and $C$ rises towards $V_{D D}$ Once $C$ reaches a threshold $C_{h i} \sim 4 V$, the state is switched to $T_{u}$.

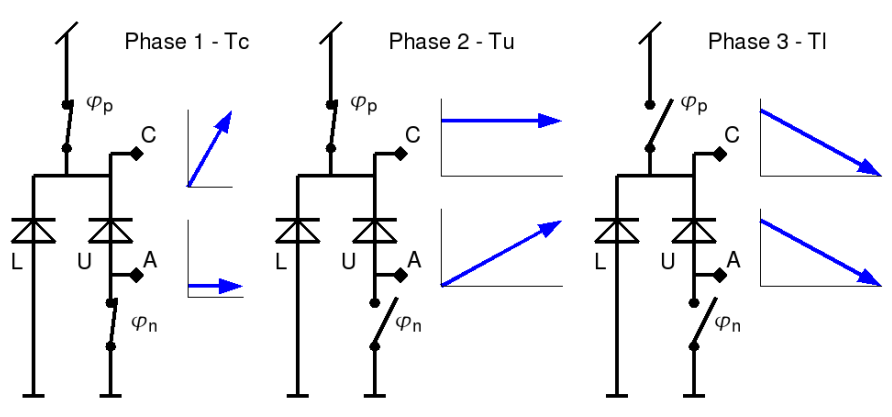

Fig. 4. Vertacolor circuit in the three states and the voltages on $A$ and $C$

2) State $T_{u}$ : Now $\varphi_{n}$ is opened and $\varphi_{p}$ stays shorted. Node $C$ is still attached to $V_{D D}$. Node $A$ is floating.

The photocurrent in $U$ starts discharging it. The photocurrent in $L$ is not relevant, because both of its nodes are attached to the rails.

The voltage of $A$ rises until $U$ is mostly discharged and $A$ reaches a threshold $A_{h i} \sim 3.8 \mathrm{~V}$. When this condition is detected the state is switched to $T_{l}$.

3) State $T_{l}$ : Because $\varphi_{p}$ is opened, both switches are now open. The photocurrent in $L$ starts to discharge it. Thus $C$ drops until $L$ is mostly discharged and $C$ reaches a threshold $C_{l o} \sim 1 V$. When this condition is detected the state is changed back to the initial state $T_{c}$.

\section{B. The entire circuit}

The entire circuit (Fig. 5) consists of the vertacolor circuit from Fig. 3 plus a control circuit that is divided into analog and digital parts.

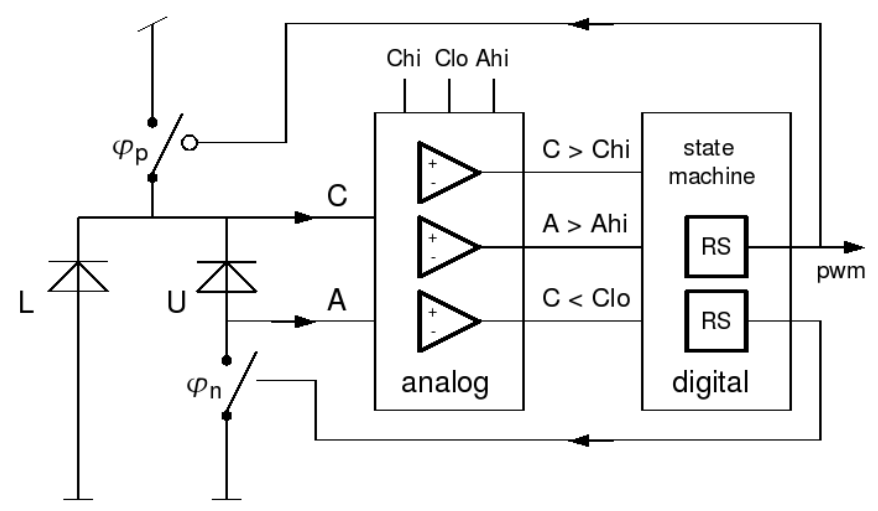

Fig. 5. Entire circuit

\section{Analog Control Part}

The analog control part consists of three 5-transistor comparators. In Fig. 5 it is shown as the middle block.

The nodes $A$ and $C$ are inputs to the analog control part. Three threshold voltages $A_{h i}, C_{l o}$ and $C_{h i}$ are also connected to the analog part.

The analog part compares the voltage at node $A$ to $A_{h i}$ and the voltage at node $C$ to $C_{l o}$ and $C_{h i}$. The three (digital) 


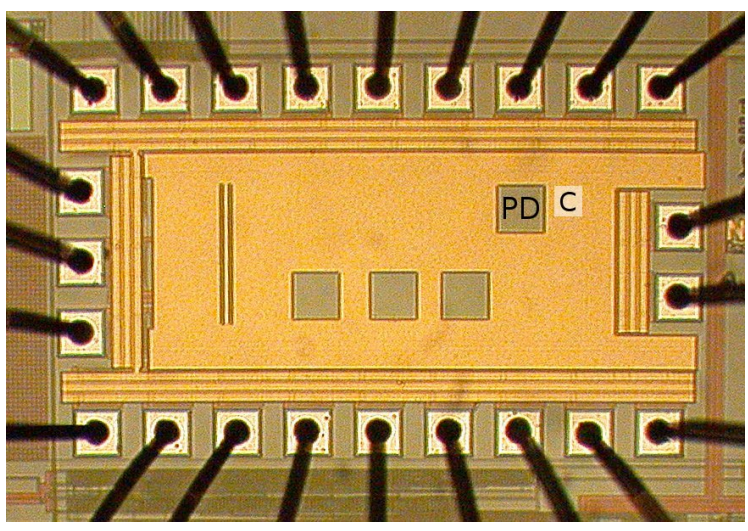

Fig. 6. die photo, $\mathrm{PD}$ is the vertacolor structure, at $\mathrm{C}$ is the control circuit

comparator output signals are fed into the digital part of the control circuit.

\section{Digital Control Part}

The digital control part has the three conditions as input and outputs the signals controlling the two switches.

First the 3 input signal are conditioned by Schmitt-triggers. Then they are fed into a state machine built from two RS flip-flops that has the following state transition table:

\begin{tabular}{lll} 
current state & transition condition & next state \\
\hline$T_{c}$ & $C>C_{h i}$ & $T_{u}$ \\
$T_{u}$ & $A>A_{h i}$ & $T_{l}$ \\
$T_{l}$ & $C<C_{l o}$ & $T_{c}$
\end{tabular}

The "current state" is held until the "transition condition" is true, then the "next state" is entered. The output signals of the state machine have already been shown in Fig. 3.

Because the state machine is built from binary logic with 2 state bits, there can also be a fourth, unwanted (parasitic) state. We need to verify what happens when this state is taken, i.e. on startup.

In the parasitic state $\varphi_{p}$ is on and $\varphi_{n}$ off. Thus both diodes discharge until $C<C_{l o}$, then the state machine goes to state $T_{c}$. Thus we cannot be stuck in the parasitic state, so there is no need for a reset or startup circuit.

\section{RESULTS}

A chip containing this circuit and other structures was built in a $0.5 \mu \mathrm{m} \mathrm{3-metal} \mathrm{2-poly} \mathrm{process} \mathrm{through} \mathrm{MOSIS}$ (Fig. 6). The area of the control circuit is about $0.007 \mathrm{~mm}^{2}$ but no attempt was made to minimize this area. Metal 3 was used to protect the circuits from parasitic photocurrents. We integrated two poly capacitors to be able to measure absolute photocurrents. They can be conditionally switched in parallel to the photodiodes, to increase the total capacitance by a known reference value.

Under indoor illumination conditions (fluorescent light) with a irradiance of about $1.7 \mathrm{~W} / \mathrm{m}^{2}$ the chip runs at a frequency of about $150 \mathrm{~Hz}$ and a duty cycle of about $25 \%$. The variabilities of the duty cycle and frequency over 5 chips are about one percent. Using color filters from an Edmund hobby
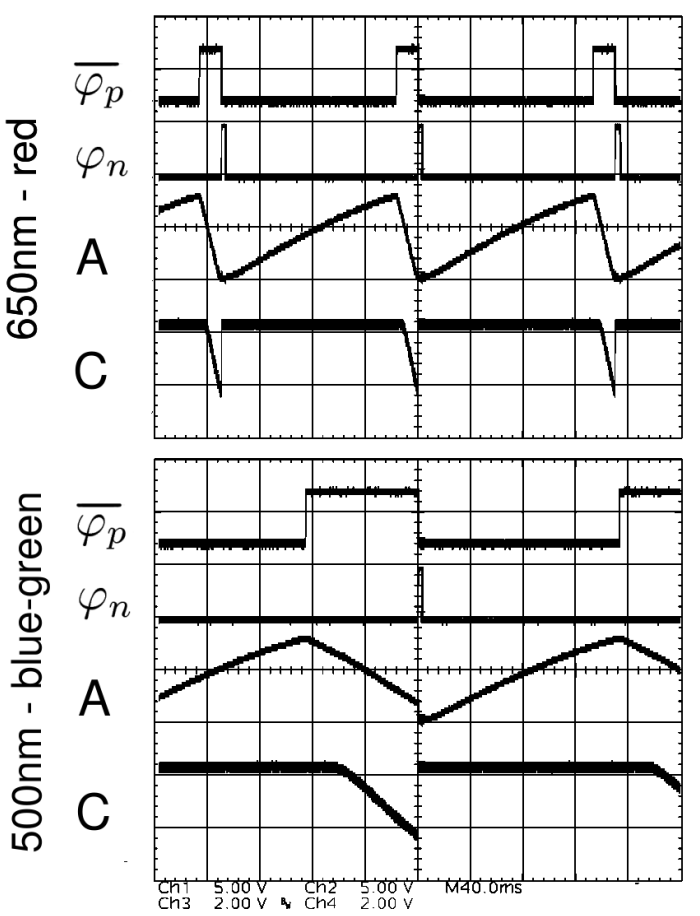

Fig. 7. Scope trace, upper at red $(650 \mathrm{~nm})$, lower at blue-green $(500 \mathrm{~nm})$

kit (to observe basic functionality) cause obvious changes in the duty cycle.

The scope traces in Fig. 7 show $\overline{\varphi_{p}}$ (inverted), $\varphi_{n}$, and the nodes A and C. Duration $T_{l}$ (when $\overline{\varphi_{p}}$ is high) varies a lot between red and blue. There is a large change in the duty cycle because of the change of photocurrent in L. Also the change of the current magnitude in $\mathrm{L}$ can directly be seen as the different slope when $\mathrm{A}$ and $\mathrm{C}$ are falling.

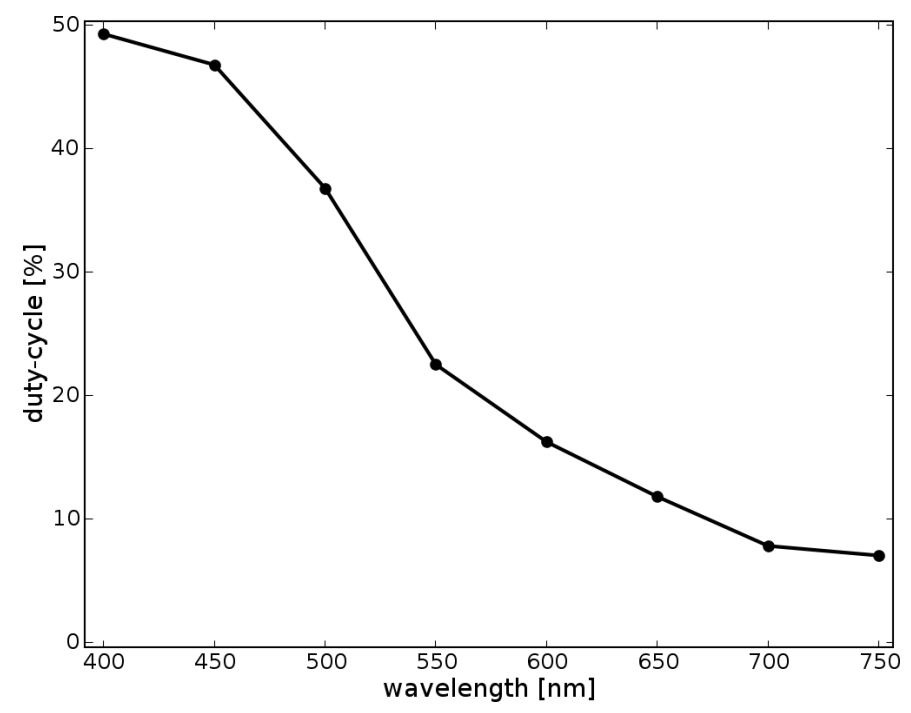

Fig. 8. Monochromator measurement: duty cycle versus wavelength. Variabilities as computed by oscilloscope are smaller than markers. 


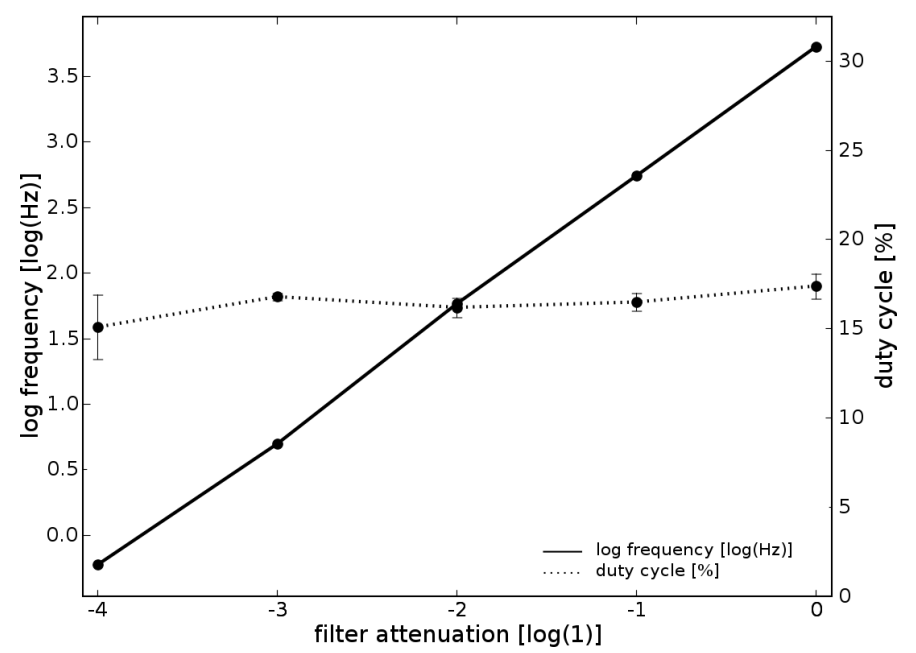

Fig. 9. Monochromator measurement: $\log$ frequency and duty cycle versus $\log$ intensity. Error bars are computed by oscilloscope.

\section{A. Spectral response characteristics}

The spectral response measurements were done using a $150 \mathrm{~W}$ fiberoptic light source with an attached monochromator (Optometrics LLC, MC1-03). The spectral width of the monochromator is $2 \mathrm{~nm}$. The monochromator was fixed over the chip so that the light output slit was about $8 \mathrm{~mm}$ above the die. Chip biases were controlled by potentiometers. The monochromator was swept over its wavelength range while measuring the circuit output duty cycle on an oscilloscope.

Fig. 8 shows that the duty cycle varies very smoothly from $50 \%$ to about $7 \%$ over the measured wavelength range of $400 \mathrm{~nm}$ to $750 \mathrm{~nm}$. This measurement is consistent with what we expected given the data in Fig. 2. The quantum efficiencies (QE) of both diodes are about the same up to $500 \mathrm{~nm}$; at $750 \mathrm{~nm}$ the QE ratio of the diodes is more than 10:1.

The output frequency should be proportional to light intensity and the duty cycle should not depend on it. To test this we used neutral density (ND) filters (Kodak Wratten \#96) along with an Edmund IR cut filter to change the light intensity without affecting the spectral shape. The IR cut filter ensures that the light falls in the specified passband of the ND filters. The measurements in Fig. 9 show that duty cycle changes only very slightly over 4 decades of illumination range.

Under room illumination conditions $\left(2 \mathrm{~W} / \mathrm{m}^{2}\right)$ the circuit draws about $4 \mu \mathrm{A}$ at $5 \mathrm{~V}$ giving a power consumption of $20 \mu \mathrm{W}$. Most of the current is consumed by the comparators in the analog control part. At this comparator bias current level, the circuit can run up to $90 \mathrm{kHz}$.

\section{CONCLUSION}

The prevalence of species with dichromatic vision and the high incidence of red-green color blindness in humans teaches that this simple spectral classification must be useful for vision.

The main achievement of this work is the realization of a simple circuit built in vanilla CMOS that turns colored light into a PWM signal, where the frequency encodes intensity and the duty cycle encodes the ratio of long to short wavelength content.

Although it is unlikely that a single pixel will solve a wide range of vision problems, it is not hard to see how a very small array (e.g. $3 \times 3$ ) could be useful.

The PWM output can also be easily interfaced to other circuits for further processing. It can be directly connected to digital circuits or the duty cycle can be converted to a voltage for analog processing by a low-pass filter.

\section{ACKNOWLEDGMENT}

We want to thank J.P. Curzan and Nova Sensors for providing silicon area. Delbruck is funded by UNI-ETH Zurich. Many thanks to Y. Wang and J.P. Curzan for proof-reading this paper.

\section{REFERENCES}

[1] T. S. Katsumi Yamamoto, private communication, 2006.

[2] Y. et al., "Apparatus for sensing the wavelength and intensity of light," U.S. Patent 54,309,604, Jan. 1, 1982.

[3] G. Gilder, The Silicon Eye. New York: Norton, 2005.

[4] R. W. Rodieck, The First Steps in Seeing. Sinauer Associates, 1998.

[5] T. Delbrück and C. Mead, "Analog VLSI phototransduction by continuous-time, adaptive, logarithmic photoreceptor circuits," in $\mathrm{Vi}$ sion Chips: Implementing vision algorithms with analog VLSI circuits, C. Koch and H. Li, Eds. Los Alamitos, CA: IEEE Computer Society Press, 1995, pp. 139-161. 